\title{
Charm semileptonic decays at LHCb
}

\author{
Adam C. S. Davis ${ }^{* \dagger}$ \\ Center for High Energy Physics \\ Tsinghua University \\ Beijing, P. R. China 100084 \\ E-mail: adavis@cern.ch
}

In these proceedings, we explore the possible reach of the LHCb dataset in the area of charm semileptonic decays. Specifically, we give prospects for the measurement of $\left|V_{c s}\right| /\left|V_{c d}\right|$ using $\mathscr{B}\left(D^{0} \rightarrow K^{-} \mu^{+} v_{\mu}\right) / \mathscr{B}\left(D^{0} \rightarrow \pi^{-} \mu^{+} v_{\mu}\right)$ with Run I data. Preliminary projections show that the LHCb Run I dataset would give a relative statistical uncertainty of $\sim 0.2 \%$ on this ratio. We also motivate the search for lepton non-universality in the charm sector.

9th International Workshop on the CKM Unitarity Triangle

28 November - 3 December 2016

Tata Institute for Fundamental Research (TIFR), Mumbai, India

* Speaker.

${ }^{\dagger}$ On behalf of the LHCb Collaboration 


\section{Introduction}

The LHCb detector at CERN[1] is a single arm forward spectrometer designed for precision studies of $b$ and $c$ hadrons in collisions at the Large Hadron Collider (LHC). During Run I of the LHC, the LHCb detector collected approximately $3 \mathrm{fb}^{-1}$ of data at $\sqrt{s}=7$ and $8 \mathrm{TeV}$, and in Run II, approximately $2 \mathrm{fb}^{-1}$ to date. Between 2011 and 2016, LHCb has reconstructed approximately 1.8 billion charm hadrons. In these proceedings, we explore the physics reach of the LHCb dataset with respect to semileptonic $D$ meson decay.

These proceedings are broken into four sections: First, a brief review of the formalism of semileptonic decays in charm, including the relevant differential decay rate. Second, we explore the experimental challenges present for LHCb and address specific concerns for neutrino reconstruction and $q^{2}$ resolution, where $q$ is the momentum transfer to the lepton and neutrino. Third, we present sensitivity estimates for the measurement of $\left|V_{c s}\right| /\left|V_{c d}\right|$ or form-factors from the ratio of decays $D^{0} \rightarrow K^{-} \mu^{+} v_{\mu}$ and $D^{0} \rightarrow \pi^{-} \mu^{+} v_{\mu}{ }^{1}$. Finally we present the motivation for the search for lepton non-universality in the charm system.

\section{Theoretical Overview}

\subsection{Differential decay rates}

The differential decay rate of the $D^{0}$ meson to a pseudoscalar final state $P$ via semileptonic decay at leading order can be written in the following well-known form[2]

$$
\begin{aligned}
\frac{d \Gamma\left(D^{0} \rightarrow P^{-} \ell^{+} v_{\ell}\right)}{d q^{2}}=\left|V_{c Q}\right|^{2} & \frac{G_{F}^{2}}{24 \pi^{3}} \frac{\left(q^{2}-m_{\ell}^{2}\right)^{2} \sqrt{E_{P}^{2}-m_{P}^{2}}}{q^{4} m_{D^{0}}^{2}} \\
\times & {\left[\left(1+\frac{m_{\ell}^{2}}{2 q^{2}}\right) m_{D^{0}}\left(E_{P}^{2}-m_{P}^{2}\right)\left|f_{+}\left(q^{2}\right)\right|^{2}+\frac{3 m_{\ell}^{2}}{8 q^{2}}\left(m_{D^{0}}^{2}-m_{P}^{2}\right)^{2}\left|f_{0}\left(q^{2}\right)\right|^{2}\right] . }
\end{aligned}
$$

Here, $Q$ represents the outgoing quark from the weak vertex, the terms $f_{+}\left(q^{2}\right)$ and $f_{0}\left(q^{2}\right)$ are the vector and scalar form factors, respectively, used to parameterize the hadronic current.

The differential decay rate measured at experiments can be broken down into two pieces of interest. First, on the right hand side of Equation 2.1 starts with CKM factors $V_{c Q}$, which are of interest in testing unitarity of the CKM matrix. Second the right hand side depends on the vector form factor $f_{+}\left(q^{2}\right)$ and scalar form factor $f_{0}\left(q^{2}\right)$. Measurements of the form factor dependence provides useful input to Lattice QCD calculations. The interesting measurements which could be made by LHCb are:

1. Measure the differential branching fractions for $D^{0} \rightarrow h^{-} \mu^{+} v_{\mu}$ as a function of $q^{2}$. This helps constrain the quantity $\left|f_{+}\left(q^{2}\right)\right|^{2}\left|V_{c Q}\right|^{2}$ and $\left|f_{0}\left(q^{2}\right)\right|^{2}\left|V_{c Q}\right|^{2}$

2. Using external constraints on the form factors from Lattice QCD, measure $\left|V_{c Q}\right|$ directly relative to a normalization channel

\footnotetext{
${ }^{1}$ Unless explicitly stated otherwise, charge-conjugate decays are implied
} 
3. Using external constraints on $\left|V_{c Q}\right|$, measure the form factor dependence, relative to a normalization channel

4. Test lepton universality using decays which differ only by the lepton in the final state

\section{Experimental Challenges}

One of the major challenges of measuring semileptonic decays at LHCb is the partially reconstructed final state. Unlike at $e^{+} e^{-}$machines, the hadron collider environment does not allow reconstruction of the missing neutrino using beam energy constraints, nor separation of the decays of interest into hemispheres. However, a host of experimental techniques exist for reconstruction of the neutrino momentum and the $q^{2}$ distributions. From kinematics alone, the neutrino momentum is completely constrained perpendicular to the $D^{0}$ flight direction. We label this transverse momentum $p_{T}^{\prime}(v)=-\left(p_{T}^{\prime}(P)+p_{T}^{\prime}(\ell)\right)$. A sketch is given in Figure 1 .

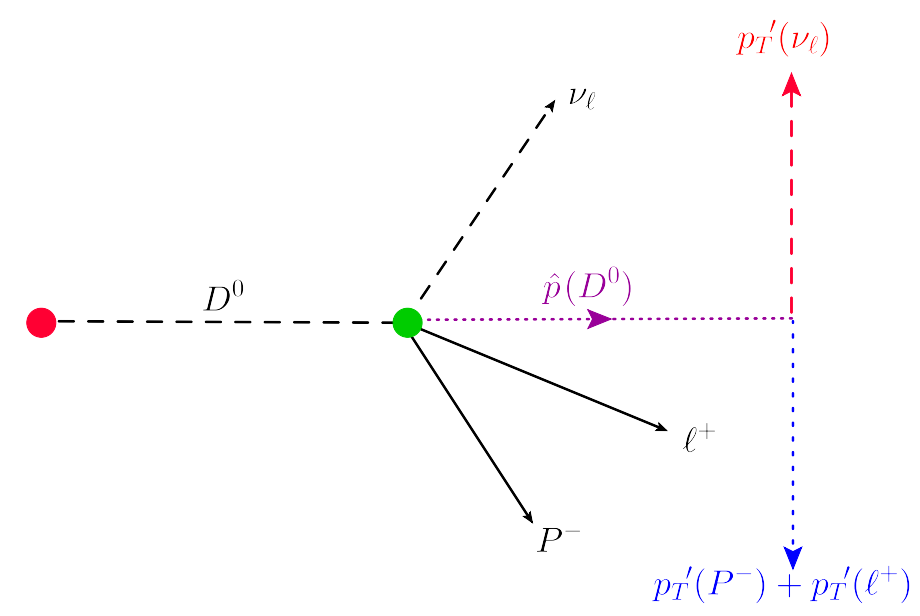

Figure 1: Cartoon of the $D^{0}$ meson semileptonic decay, with the definition of the momentum component $p_{T}^{\prime}(v)$. The red and green dots represent the origin and decay vertex of the $D^{0}$, respectively, the arrowed black lines represent the momentum components of the final state particles, the purple line indicates the initial flight direction of the $D^{0}$, and the red and blue dashed lines represent the component of the momentum $p_{T}^{\prime}$ perpendicular to the $D^{0}$ flight direction.

Using energy and momentum conservation to solve for the remaining component of the neutrino momentum leaves a two-fold ambiguity. While possible, simply choosing one of the solutions of the neutrino momentum can lead to biases in the distributions of interest, specifically with respect to $q^{2}$. There are several well-known methods for calculating the missing neutrino momentum component, relying on a variety of different techniques:

- $k$-factor method: Using simulated events, determine the factor $k$ as a function of visible daughter mass which predicts the true $D^{0}$ momentum. Such an approach usually determines an average value $\langle k\rangle$ written as $p\left(D^{0}\right)=\frac{p(K \ell)}{\langle k(m(K \ell))\rangle}$. This approach has been used previously in many analyses, one such example being the measurement of the $B^{0}$ meson oscillation frequency $\Delta m_{d}[3]$. 
- Corrected $D^{0}$ mass: One can use as an approximation to the true $D^{0}$ mass the quantity $m_{\text {Corrected }}=\sqrt{m^{2}(K \ell)+\left|p_{T}^{\prime}\right|^{2}}+p_{T}^{\prime}$. This quantity has the usefulness that it will peak at the nominal mother mass for true decays and have a long tail that extends to lower $m_{\text {corrected }}$. Decays of multibody final states will then peak more strongly towards lower $m_{\text {corrected }}$ allowing for good separation between signal and background. Such a method has been used successfully in the measurement of $\left|V_{u b}\right|$ from $\Lambda_{b} \rightarrow p \mu \nu$ decays [4].

- Cone-closure: By enforcing that the $D^{0}$ be the daughter of a $D^{*+} \rightarrow D^{0} \pi^{+}$decay, the additional mass constraint of the $D^{*+}$ breaks the ambiguity of the momentum of the neutrino. The method relies on the fact that in the $K \ell$ rest frame, $p\left(D^{0}\right)=p(v)$, and the slow pion forms the axis of a cone around which the neutrino momentum lies. The solution of the momentum is then the one that most closely aligns the $D^{0}$ momentum to the $D^{0}$ flight direction. Such a procedure was used in the E687 experiment at Fermilab [5].

- Recently, a new method using multivariate regression to help choose the correct neutrino momentum of $b$-hadron has been presented [6]. While the use case presented is for $b$-hadron decays, the algorithm should be easily extendible to $c$-hadron decays.

Each of these methods has its shortcomings, but provides an estimate of the momentum of the $D^{0}$ and thus the calculation of $q^{2}$.

\section{Sensitivity for CKM matrix element measurements}

As an example of an accessible measurement at $\mathrm{LHCb}$, we estimate the sensitivity of the measurement of the ratio of CKM matrix elements $\left|V_{c s}\right| /\left|V_{c d}\right|$. The measurement can be made by comparing the branching ratios of $D^{*+} \rightarrow D^{0} \pi$ with $D^{0} \rightarrow K^{-} \mu^{+} v_{m} u$ and $D^{0} \rightarrow \pi^{-} \mu^{+} v_{\mu}$. Such a measurement is tractable at LHCb due to the high efficiency of reconstructing muons. By enforcing that the $D^{0}$ originate from a $D^{*+}$ decay, the $q^{2}$ dependence of both channels can be made unambiguously. This is a very similar measurement to the measurement in [4]. The advantage of such a ratio measurement is that the majority of the trigger, selection and detection efficiencies cancel in the ratio. Additionally, the corrected mass can provide a stable handle on missing neutral backgrounds.

The sensitivity estimate can be made in the following way: as the branching ratio of the decay $D^{\rightarrow} K^{-} \mu^{+} v_{\mu}$ is similar to that of $D^{0} \rightarrow K^{-} \pi^{+}$, an initial estimate of yields can be made for the Run I dataset by taking number of $D^{0} \rightarrow K^{-} \pi^{+}$decays from the CPV search in charm using the same decay [7]. This gives an initial estimate of $\sim 56$ million $D^{0} \rightarrow K^{-} \mu^{+} v_{\mu}$ decays, and roughly one order of magnitude fewer decays of $D^{0} \rightarrow \pi^{-} \mu^{+} v_{\mu}$ decays. The software trigger to use for such a decay at the LHCb experiment is one which searches for inclusive $D^{*+} \rightarrow D^{0} \pi^{+}$decays. Such a trigger was only operational during two-thirds of the 2012 Run I operation, limiting the statistics to roughly $60 \%$ of the nominal value. Assuming that the remaining efficiency differences are at the order of $20 \%$, as the major differences will be reconstructing the muon and forming a good vertex, this leaves roughly 4.4 million signal candidates. This yield would directly relate to a relative systematic uncertainty of $0.2 \%$. 
These numbers are not simply out of thin air. A validation of the tracking efficiency in the analysis of $a_{s l}^{s}[8]$ used the sample $D^{0} \rightarrow K^{-} \mu^{+} v_{\mu}$ to calculate the tracking asymmetry of the muonpion pair. With loose selections, the analysis reconstructed $5 M$ signal candidates. The total fit was performed simultaneously between $D^{0} \rightarrow K^{-} \mu^{+} v_{\mu}$ and $\bar{D}^{0} \rightarrow K^{+} \mu^{-} \bar{v}_{\mu}$ samples in individual bins of visible mass $m(K \mu)$, with the signal shape being derived empirically. An example plot of the visible mass difference $m(K \mu \pi)-m(K \mu)$ for the 2012 dataset using the magnet down polarity fit in the range of visible mass $1600 \leq m(K \mu)<1700 \mathrm{MeV} / c^{2}$ is shown in Figure 2.

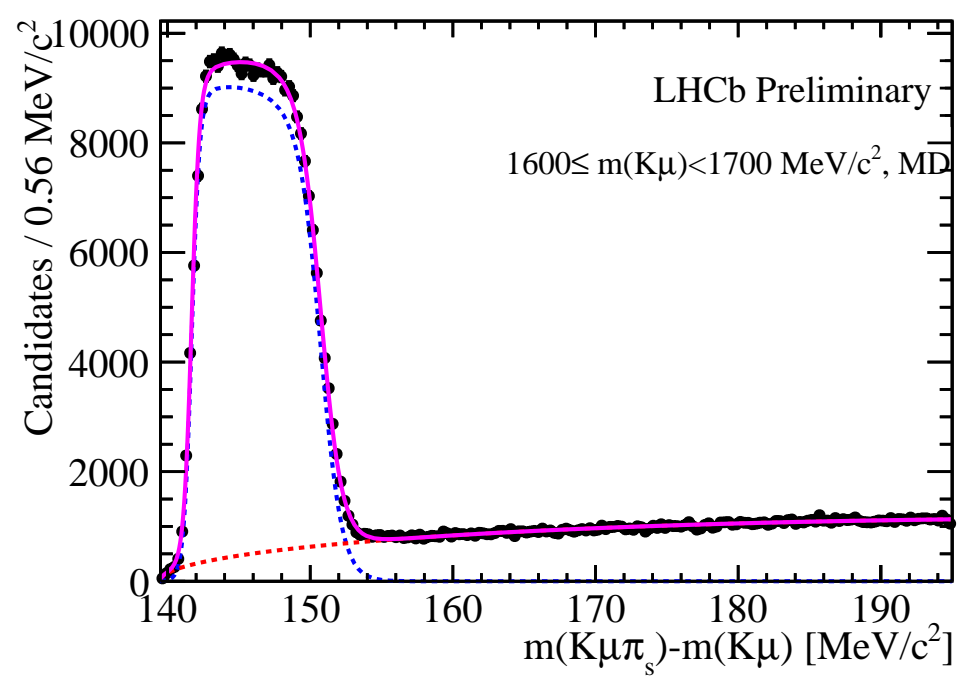

Figure 2: Fit to the visible difference in mass $m(K \mu \pi)-m(K \mu)$ of the decay $D^{0} \rightarrow K \mu v$. The points correspond to the sample of $D^{0} \rightarrow K \mu v$ decays in the 2012 magnet down dataset lying in the mass range $1600 \leq m(K \mu)<1700 \mathrm{MeV} / c^{2}$. The total fit is shown in magenta, with the signal component shown in dashed blue, and the combinatorial background in dashed red.

\section{Lepton non-universality in semileptonic $D$ decays}

Lepton non-universality has been a hot topic in the past few years mainly driven by possible anomalies in the semileptonic decays of $B \rightarrow D^{*} \ell v$ with a $\tau$ in the final state compared to a $\mu$, as well as possible differences seen in decays of the form $b \rightarrow s \ell \ell$. Such tensions with the standard model beg the question whether or not there is a similar measurement to be made in other modes. No such measurement of lepton non-universality has been made in the charm sector, but each of the individual relevant branching ratios has been measured [9]. By taking the simple ratio of relevant decays, and comparing with the $q^{2}$ integrated standard model prediction from [10] shows a consistent trend towards higher ratios than expected. This is illustrated in Figure 3.

All of the modes presented in Figure 3 are worth pursuing. Recent theory activity [10] shows that the measurement of the ratio $\mathscr{B}\left(D^{0} \rightarrow K^{-} e^{+} v_{e}\right) / \mathscr{B}\left(D^{0} \rightarrow K^{-} \mu^{+} v_{\mu}\right)$, for example, specifically as a function of $q^{2}$, allows for a direct probe of current bounds on allowed scalar Wilson coefficients. By using the same statistics quoted above for $D^{0} \rightarrow K^{-} \ell^{+} v_{\ell}$, the measurement of the ratio of the electron to muon modes would reduce the error on the bottom point in Figure 3 by an order 


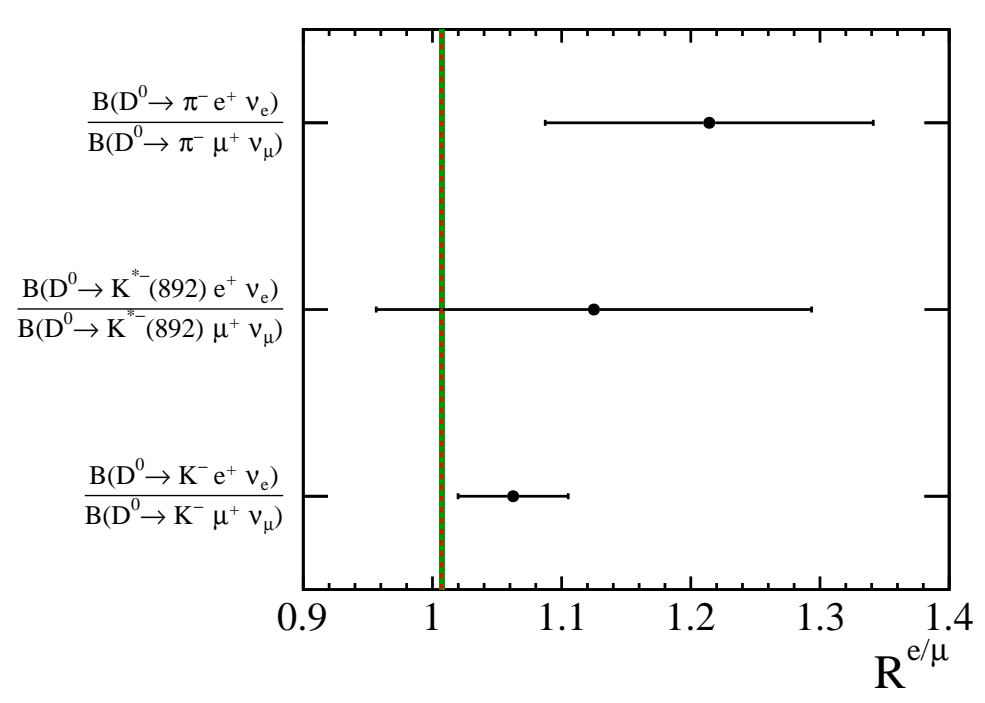

Figure 3: Comparison of the ratio of measured branching fractions for $D^{0} \rightarrow h^{-} e^{+} v$ and $D^{0} \rightarrow h^{-} \mu^{+} v$. The term $h$ denotes the hadron in question, either $K^{-}, \pi^{-}$or $K^{*-}(892)$. Each point represents the ratio from the measurements reported in [9] compared to the standard mode prediction (red dashed line) and its error(green band) provided by S. Fajfer using [11]. The central values of each point lie systematically to one side of the standard model prediction.

of magnitude. While the measurement of the electron mode is certainly difficult at $\mathrm{LHCb}$, due to the fact that bremsstrahlung recovery is not easy, and the measurement of the tracking efficiencies of the electron candidate is non-trivial, the measurement is not impossible; LHCb has already measured many channels with electrons, including the angular analysis of $B^{0} \rightarrow K^{* 0} e^{+} e^{-}[12]$ and the search for lepton flavor violation in the decay of $D^{0} \rightarrow e^{ \pm} \mu^{\mp}[13]$.

\section{Conclusion}

We present preliminary estimates on the reach of LHCb in the field of semileptonic $D$ meson decay. We find that a measurement of $\left|V_{c s}\right| /\left|V_{c d}\right|$ would give a relative statistical uncertainty of $\sim 0.2 \%$ using the Run I dataset. We also motivate the first search for lepton non-universality in the charm sector. It is important to note that all estimates are using the Run I dataset, and LHCb continues to take its Run II dataset with more statistics and improved triggering strategies.

\section{References}

[1] R. Aaij et al. [LHCb Collaboration], Int. J. Mod. Phys. A 30, no. 07, 1530022 (2015) doi:10.1142/S0217751X15300227 [arXiv:1412.6352 [hep-ex]].

[2] S. Aoki et al., Eur. Phys. J. C 77, no. 2, 112 (2017) doi:10.1140/epjc/s10052-016-4509-7 [arXiv:1607.00299 [hep-lat]].

[3] R. Aaij et al. [LHCb Collaboration], Eur. Phys. J. C 76, no. 7, 412 (2016) doi:10.1140/epjc/s10052-016-4250-2 [arXiv:1604.03475 [hep-ex]]. 
[4] R. Aaij et al. [LHCb Collaboration], Nature Phys. 11, 743 (2015) doi:10.1038/nphys3415 [arXiv:1504.01568 [hep-ex]].

[5] W. E. Johns, FERMILAB-THESIS-1995-05, UMI-96-02371.

[6] G. Ciezarek, A. Lupato, M. Rotondo and M. Vesterinen, JHEP 1702, 021 (2017) doi:10.1007/JHEP02(2017)021 [arXiv:1611.08522 [hep-ex]].

[7] R. Aaij et al. [LHCb Collaboration], Phys. Rev. Lett. 111, no. 25, 251801 (2013) doi:10.1103/PhysRevLett.111.251801 [arXiv:1309.6534 [hep-ex]].

[8] R. Aaij et al. [LHCb Collaboration], Phys. Rev. Lett. 117, no. 6, 061803 (2016) doi:10.1103/PhysRevLett.117.061803 [arXiv:1605.09768 [hep-ex]].

[9] C. Patrignani et al. [Particle Data Group], Chin. Phys. C 40, no. 10, 100001 (2016). doi:10.1088/1674-1137/40/10/100001

[10] S. Fajfer, I. Nisandzic and U. Rojec, Phys. Rev. D 91, no. 9, 094009 (2015) doi:10.1103/PhysRevD.91.094009 [arXiv:1502.07488 [hep-ph]].

[11] J. Koponen, C. T. H. Davies, G. C. Donald, E. Follana, G. P. Lepage, H. Na and J. Shigemitsu, arXiv:1305.1462 [hep-lat].

[12] R. Aaij et al. [LHCb Collaboration], JHEP 1504, 064 (2015) doi:10.1007/JHEP04(2015)064 [arXiv:1501.03038 [hep-ex]].

[13] R. Aaij et al. [LHCb Collaboration], Phys. Lett. B 754, 167 (2016) doi:10.1016/j.physletb.2016.01.029 [arXiv:1512.00322 [hep-ex]]. 